\title{
Pilot Sequence Assignment for Spatially Correlated Massive MIMO Circumstances
}

\author{
Pengxiang $\mathrm{Li}^{{ }^{*}}$, Yuehong Gao ${ }^{1}$, Zhidu $\mathrm{Li}^{1}$, Dacheng Yang ${ }^{1}$ \\ ${ }^{1}$ Wireless Theories and Technologies Lab, Beijing University of Posts and Telecommunications \\ Beijing 100876, China \\ [e-mail: lpengxiang@163.com; yhgao@bupt.edu.cn; prclzd@126.com; yangdc@bupt.edu.cn] \\ *Corresponding author: Pengxiang Li
}

Received January 25, 2018; revised April 12, 2018; accepted May 15, 2018;

published January 31, 2019

\begin{abstract}
For massive multiple-input multiple-output (MIMO) circumstances with time division duplex (TDD) protocol, pilot contamination becomes one of main system performance bottlenecks. This paper proposes an uplink pilot sequence assignment to alleviate this problem for spatially correlated massive MIMO circumstances. Firstly, a single-cell TDD massive MIMO model with multiple terminals in the cell is established. Then a spatial correlation between two channel response vectors is established by the large-scale fading variables and the angle of arrival (AOA) span with an infinite number of base station (BS) antennas. With this spatially correlated channel model, the expression for the achievable system capacity is derived. To optimize the achievable system capacity, a problem regarding uplink pilot assignment is proposed. In view of the exponential complexity of the exhaustive search approach, a pilot assignment algorithm corresponding to the distinct channel AOA intervals is proposed to approach the optimization solution. In addition, simulation results prove that the main pilot assignment algorithm in this paper can obtain a noticeable performance gain with limited BS antennas.
\end{abstract}

Keywords: Achievable system capacity, uplink pilot sequence assignment, single-cell TDD massive MIMO, spatially correlated channels

The research is supported by National Science and Technology Major Project of the Ministry of Science and Technology, and the project name is "Evaluation and verification of candidate solutions for international standardization of 5G” ' with grant No. 2018ZX03001024-006. 


\section{Introduction}

Today most of the wireless communication systems are deployed in microwave frequencies. Therefore, lower frequency bands are becoming more and more congested. However, the available spectrum resource in centimeter-wave (cm-wave) bands and millimeter-wave (mm-wave) bands has not been fully developed [1]. Compared with the microwave propagation channels, the cm-wave propagation channels and the mm-wave propagation channels have fundamentally different channel characteristics. Especially, the adoption of the higher frequency bands will incur a much more severe propagation loss even in the free space. This makes them not adequate in long-distance communication circumstances.

One solution would be to adopt extensive antennas at base stations (BSs), which is called massive multiple-input multiple-output (MIMO) technology. In fact, the use of higher frequency bands makes this possible. The number of BS antennas under consideration varies from 256 to 1024 when the mm-wave frequencies are adopted [2]. With the large scale antenna arrays, massive MIMO technology can concentrate the BS energy into the target directions and provide a highly desired array gain to compensate the disappointing propagation loss. Besides the coverage enhancement, massive MIMO technology also works well in improving the date rate and the energy efficiency [3]. It has become a major research direction of the 5-th generation (5G) mobile networks. Specifically, the basic overviews of massive MIMO networks, including the challenges and the opportunities, was presented in detail [4].

Channel state information (CSI) is very important for massive MIMO transmissions to meet a good system performance. In massive MIMO circumstances with time division duplex (TDD) protocol, channel reciprocity can be used [5]. And CSI at the BSs is estimated by adopting the designated pilot signals that are periodically transmitted at all terminals in the system. However, the orthogonal pilot assignment cannot be carried out in most cases. When one pilot sequence is reused by several terminas in the system, pilot contamination problem emerges [6]. With the increase of BS antenna number, pilot contamination becomes one of the main system performance bottlenecks. In massive MIMO circumstances with frequency division duplex (FDD) protocol, channel reciprocity cannot be used anymore. And uplink feedback is on the agenda to estimate CSI at the BSs. However, the amount of the feedback information from the terminals is linearly correlated with the amount of transmit antennas at the BSs. Therefore, the key issue is how to optimize the feedback mode [7].

A significant number of papers are dedicated to mitigating pilot contamination. For example, the interference of pilot sequence and the channel quality of terminal are considered together to mitigate pilot contamination [8]. Pilot sequences and data sequences are superimposed over the entire frame in massive MIMO circumstances and the corresponding channel estimation scheme was analyzed [9]. In addition, a projection approach which does not require the pilot signals to find the appropriate subspaces was proposed [10]. Together with the power control algorithm, it can effectively mitigate pilot contamination.

Note that most of the papers mentioned above adopt the independent and identically distributed fading model. However, the practical wireless channels are not always so ideal. The spatial correlation property should be studied to further exploit the potential of massive MIMO technology. A coordination approach was proposed by using the second-order information among terminals to alleviate pilot contamination for spatially correlated massive MIMO scenes [11]. A correlation between two channel characteristics, spatial correlations and power angle spectrum, was established [12]. Subsequently, the expressions for BS receivers 
and BS precoders were developed to optimize the detection error for spatially correlated massive MIMO channels.

This paper studies the pilot sequence assignment for spatially correlated massive MIMO circumstances. Specifically, we have

- In [11] and [12], the orthogonality between two terminals was defined by the angle between their channel covariance matrices. Therefore, the individual covariance matrices of all users in the system should be calculated. However, this will take too much computing resources if the amount of BS antennas is large. Specifically, a spatial correlation between two channel response vectors is established by the large-scale fading variables and the angle of arrival (AOA) span when the amount of BS antennas tends to infinity. Then the complex process of calculating covariance matrices could be omitted. And the corresponding expression for the achievable system capacity is derived.

- The pilot assignment approaches mentioned in [11] and [12] indicated that the terminals with orthogonal channel covariance matrices can reuse the same pilot sequences. However, they certainly do not consider the different channel qualities. In this paper, a problem is designed to optimize the achievable system capacity and a low-complexity pilot assignment algorithm is proposed to approach the optimization solution. Specifically, after the terminals with high AOA similarities are assigned with the orthogonal pilot sequences, each unhandled terminal is matched up with the most "appropriate" pilot sequence according to the AOA span in a sequential way. Simulation results prove that this pilot assignment algorithm can obtain a noticeable performance gain with limited BS antennas.

In this paper, section 2 reveals the massive MIMO system and the expression for the achievable system capacity. Section 3 reveals the optimization problem and the proposed pilot assignment algorithm. Section 4 reveals simulation results. Section 5 is a summary.

In addition, scalar is denoted by italics. Vector (matrix) is denoted by boldface lowercase (uppercase). The complex number is denoted by $C$. The transpose is denoted by $(\cdot)^{\mathrm{T}}$. The Hermitian transpose is denoted by $(\cdot)^{\mathrm{H}} \cdot \mathrm{e}^{\mathrm{x}}$ is denoted by $\exp (x)$. The circularly symmetric complex Gaussian variable with mean 0 and variance 1 is denoted by $C N(0,1)$. The empty set is denoted by $\phi$. The absolute value is denoted by $\mid \cdot$.

\section{System Model}

Firstly, a single-cell TDD massive MIMO model with multiple terminals in the cell is presented. And a spatial correlation between two channel response vectors is established when the BS antenna number is equal to infinity. Then the corresponding expression for the achievable system capacity is derived.

\subsection{System Model}

As presented in Fig. 1, the radius of this massive MIMO cell is $R$. The BS with its central position is configured with $M$ independent antennas. Moreover, $K$ single-antenna terminals are randomly located without the radius of $r$. In addition, the terminal-cooperation which leads to a substantial side-information overhead is not invoked there. Specifically, all transmissions and receptions in this massive MIMO cell are assumed to be synchronous. In this case, the intra-cell interference will be maximized. Therefore, this assumption means the worst possible case [6][11]. 


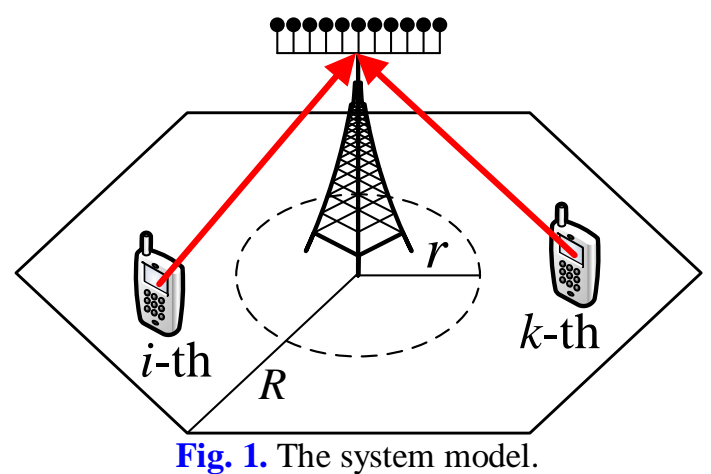

\subsection{Spatially Correlated Channel Model}

We adopt the ray-tracing based channel model given in [12]. Next, we assume that the channel propagation vector between BS antennas and the $k$-th user in the cell $\mathbf{h}_{k} \in C^{M \times 1}$ can be denoted by

$$
\mathbf{h}_{k}=\sqrt{\beta_{k}} \int_{\theta_{k}^{\min }}^{\theta_{\text {max }}^{\max }} \mathbf{v}\left(\theta_{k}\right) d \theta_{k}
$$

In (1), $\beta_{k}$ means the average large-scale fading coefficient between BS antennas and the corresponding user. In practical wireless communication networks, such as the long term evolution (LTE) networks, a user connects to the BS which has the best channel quality firstly. In the same time, it can still receive the signals transmitted by other BSs because the switching process among different cells is likely to happen at any time with the movement of this terminal [13][14]. In addition, we can think that the average large-scale fading variables mentioned above can be easily obtained by the BS because they change very slowly in reality [6][15]. Moreover, for the $k$-th user in the cell, $\left[\theta_{k}^{\min }, \theta_{k}^{\max }\right]$ denotes the channel AOA interval obtained at the BS. In addition, $\mathbf{v}(\theta) \in C^{M \times 1}$ denotes the BS steering vector related to the incidence direction $\theta$. Specifically, we set $\theta \in\left[-\frac{\pi}{2}, \frac{\pi}{2}\right]$ in this paper. This can be achieved by adopting the directional antenna arrays at the BS. If uniform linear array (ULA) is installed at the BS and the antenna spacing equals to a half wavelength distance, $\mathbf{v}(\theta)$ can be expressed as

$$
\mathbf{v}(\theta)=[1, \exp (-j \pi \sin \theta), \exp (-j \pi 2 \sin \theta), \cdots, \exp (-j \pi(M-1) \sin \theta)]^{\mathrm{T}} \text {.(2) }
$$

Theorem 1: When the amount of BS antennas is equal to infinity, the BS steering vectors associated with distinct incidence angles are orthogonal. And this theorem can be formulated as

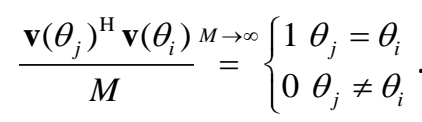

Proof: Please see [11].

Then the spatial correlation between two channel response vectors is established as

Based on Theorem 1, we have

$$
\frac{\mathbf{h}_{j}^{\mathrm{H}} \mathbf{h}_{i}}{M}=\sqrt{\beta_{j} \beta_{i}} \int_{\theta_{j}^{\min }}^{\theta_{\theta_{i}^{\max }}^{\theta_{i \operatorname{mix}}^{\max }}} \frac{\mathbf{v}\left(\theta_{j}\right)^{\mathrm{H}} \mathbf{v}\left(\theta_{i}\right)}{M} d \theta_{j} d \theta_{i} \cdot(4)
$$

$$
\frac{\mathbf{h}_{j}^{\mathrm{H}} \mathbf{h}_{i}}{M} \stackrel{M \rightarrow \infty}{=}\left\{\begin{array}{ll}
0 & \text { case } 1 \\
\sqrt{\beta_{j} \beta_{i}} \theta_{j i} & \text { case } 2
\end{array} .\right.
$$

In (5), “case 1 " denotes that the AOA for the $j$-th terminal and the AOA for the $i$-th terminal 
are non-overlapping, i.e., $\left[\theta_{j}^{\min }, \theta_{j}^{\max }\right] \cap\left[\theta_{i}^{\min }, \theta_{i}^{\max }\right]=\phi$. In such situation, the two corresponding channel propagation vectors can be considered orthogonal. On the other hand, "case 2" denotes that $\left[\theta_{j}^{\min }, \theta_{j}^{\max }\right] \cap\left[\theta_{i}^{\min }, \theta_{i}^{\max }\right] \neq \phi$. Specifically, $\theta_{j i}$ denotes the span of the AOA intersection. In this case, both the average large-scale fading coefficients and the AOA span affect the spatial correlation. Compared with the correlation measurements mentioned in [11] and [12], (5) is much more intuitive because the complex process of calculating the channel covariance matrices can be omitted.

\subsection{Achievable System Capacity}

We assume that this massive MIMO cell adopts the TDD protocol and the block fading protocol. This means channel propagation vectors are going to stay the same during the entire block interval. In this paper, we assume that the length of coherence interval is $T$ orthogonal frequency division multiplexing (OFDM) symbols. In addition, coherence interval contains two parts. The first one is pilot transmission part and the length of it is $\tau$ symbols. The second one is data transmission part and the length of it is $(T-\tau)$ symbols. Next, we discuss them respectively.

For the sake of simplicity, we hold the opinion that the number of available orthogonal pilot sequences is $T$. During the first part, all the terminals in this cell transmit their own pilot signals to the only BS synchronously. And the received pilot sequences $\mathbf{Y} \in C^{M \times \tau}$ can be expressed as

$$
\mathbf{Y}=\sqrt{\rho_{k}^{\mathrm{p}}} \mathbf{h}_{k} \mathbf{z}_{k}+\sum_{i \neq k}^{K} \sqrt{\rho_{i}^{\mathrm{p}}} \mathbf{h}_{i} \mathbf{z}_{i}+\mathbf{N}_{\mathrm{p}} \cdot(6)
$$

In (6), $\rho_{k}^{\mathrm{p}}$ denotes the $k$-th user's transmission power for pilot signals. $\mathbf{z}_{k} \in C^{1 \times \tau}$ means the pilot sequence used by the $k$-th user. Specifically, we assume that $\mathbf{z}_{i} \mathbf{z}_{j}^{\mathrm{H}}=\left\{\begin{array}{l}0 i \neq j \\ 1 i=j\end{array}\right.$, for $i, j=1,2, \cdots, \tau . \mathbf{N}_{\mathrm{p}} \in C^{M \times \tau}$ means the additive pilot noise. In addition, we assume that every element in $\mathbf{N}_{\mathrm{p}}$ is $C N(0,1)$.

Next, channel estimation is implemented at the BS based on the received pilot signals. We adopt the least squares (LS) channel estimation given in [6], and the corresponding estimation result $\hat{\mathbf{h}}_{k} \in C^{M \times 1}$ can be directly obtained by associating $\mathbf{Y}$ with $\mathbf{z}_{k}^{\mathrm{H}}$ as

$$
\hat{\mathbf{h}}_{k}=\mathbf{Y} \mathbf{z}_{k}^{\mathrm{H}}=\sqrt{\rho_{k}^{\mathrm{p}}} \mathbf{h}_{k}+\sum_{i \in \Gamma_{k}} \sqrt{\rho_{i}^{\mathrm{p}}} \mathbf{h}_{i}+\mathbf{N}_{\mathrm{p}} \mathbf{z}_{k}^{\mathrm{H}} \cdot(7)
$$

In (7), the first item of the expansion reveals the desired channel propagation vector for the $k$-th terminal in the cell. In addition, the terminals in the set $\Gamma_{k}$ use the same pilot signal as that allocated to the $k$-th user, and the second item reveals pilot contamination. Moreover, the third item denotes the equivalent pilot noise. With sufficient orthogonal pilot sequences (i.e., $\tau \geq K$ ), it is obvious that $\Gamma_{k} \in \phi$. The channel estimation $\hat{\mathbf{h}}_{k}$ will only be destroyed by the equivalent pilot noise, and it can be expressed as $\hat{\mathbf{h}}_{k}^{\text {noint }}=\sqrt{\rho_{k}^{\mathrm{p}}} \mathbf{h}_{k}+\mathbf{N}_{\mathrm{p}} \mathbf{z}_{k}^{\mathrm{H}}$.

During the second part, all the terminals in this cell transmit their own data sequences to the BS synchronously. And the received data signals $\mathbf{R} \in C^{M \times \kappa}$ is denoted by

$$
\mathbf{R}=\sqrt{\rho_{k}^{\mathrm{d}}} \mathbf{h}_{k} \mathbf{x}_{k}+\sum_{j \neq k}^{K} \sqrt{\rho_{j}^{\mathrm{d}}} \mathbf{h}_{j} \mathbf{x}_{j}+\mathbf{N}_{\mathrm{d}} .(8)
$$


In (8), $\rho_{k}^{\mathrm{d}}$ denotes the $k$-th user's transmission power for data signals. $\mathbf{x}_{k} \in C^{1 \times \kappa}(\kappa=T-\tau)$ denotes the data sequence form the $k$-th terminal. $\mathbf{N}_{d} \in C^{M \times \kappa}$ denotes the additive data noise matrix. In addition, we assume that every element in $\mathbf{N}_{\mathrm{d}}$ is $C N(0,1)$.

Next, the obtained data signals is detected at the BS based on the channel estimation result in the first part. Specifically, the standard maximal ratio combining (MRC) protocol mentioned in [8] is adopted there, and the detected data sequence form the $k$-th terminal $\hat{\mathbf{x}}_{k} \in C^{1 \times \kappa}$ can be directly obtained by correlating the channel estimation result $\hat{\mathbf{h}}_{k}^{\mathrm{H}}$ with the received data signals $\mathbf{R}$ as (9a).

$$
\begin{aligned}
\hat{\mathbf{x}}_{k} & =\hat{\mathbf{h}}_{k}^{\mathrm{H}} \mathbf{R}^{(\mathrm{a})}=\underbrace{\sqrt{\rho_{k}^{\mathrm{d}}} \hat{\mathbf{h}}_{k}^{\mathrm{H}} \mathbf{h}_{k} \mathbf{x}_{k}}_{\Lambda_{1}}+\underbrace{\sum_{j \neq k}^{K} \sqrt{\rho_{j}^{\mathrm{d}}} \hat{\mathbf{h}}_{k}^{\mathrm{H}} \mathbf{h}_{j} \mathbf{x}_{j}}_{\Lambda_{2}}+\underbrace{\hat{\mathbf{h}}_{k}^{\mathrm{H}} \mathbf{N}_{\mathrm{d}}}_{\Lambda_{3}} \\
& \stackrel{(\mathrm{b})}{=} \underbrace{\sqrt{\rho_{k}^{\mathrm{p}} \rho_{k}^{\mathrm{d}}} \mathbf{h}_{k}^{\mathrm{H}} \mathbf{h}_{k} \mathbf{x}_{k}}_{\Delta_{1}}+\underbrace{\sum_{j \neq k}^{K} \sqrt{\rho_{k}^{\mathrm{p}} \rho_{j}^{\mathrm{d}}} \mathbf{h}_{k}^{\mathrm{H}} \mathbf{h}_{j} \mathbf{x}_{j}}_{\Delta_{2}}+\underbrace{\sum_{i \in \Gamma_{k}} \sqrt{\rho_{i}^{\mathrm{p}} \rho_{k}^{\mathrm{d}}} \mathbf{h}_{i}^{\mathrm{H}} \mathbf{h}_{k} \mathbf{x}_{k}}_{\Delta_{3}}+\underbrace{\sum_{i \in \Gamma_{k}} \sum_{j \neq k}^{K} \sqrt{\rho_{i}^{\mathrm{p}} \rho_{j}^{\mathrm{d}}} \mathbf{h}_{i}^{\mathrm{H}} \mathbf{h}_{j} \mathbf{x}_{j}}_{\Delta_{4}}+\mathbf{n}
\end{aligned}
$$

In (9a), $\Lambda_{1}$ denotes the desired data sequence. $\Lambda_{2}$ reveals the intra-cell interference. And $\Lambda_{3}$ denotes the equivalent data noise. Consider the fact that the BS only knows the estimations of the channels, the $k$-th user's uplink signal-to-interference and noise ratio (SINR) can be expressed as

$$
\operatorname{SINR}_{k}^{\mathrm{ach}}=\frac{\left|\sqrt{\rho_{k}^{\mathrm{d}}} \hat{\mathbf{h}}_{k}^{\mathrm{H}} \mathbf{h}_{k}\right|^{2}}{\sum_{j \neq k}^{K}\left|\sqrt{\rho_{j}^{\mathrm{d}}} \hat{\mathbf{h}}_{k}^{\mathrm{H}} \mathbf{h}_{j}\right|^{2}+\left|\hat{\mathbf{h}}_{k}^{\mathrm{H}} \mathbf{N}_{\mathrm{d}} \mathbf{N}_{\mathrm{d}}^{\mathrm{H}} \hat{\mathbf{h}}_{k}\right|} \cdot(10)
$$

Then the $k$-th user's achievable capacity is denoted by $\mathrm{C}_{k}^{\text {ach }}=W \frac{(T-\tau)}{T} \log _{2}\left(1+\operatorname{SINR}_{k}^{\text {ach }}\right)$, where $W$ denotes the total bandwidth. And the achievable system capacity can be expressed as $\mathrm{C}_{\text {total }}^{\text {ach }}=\sum_{k=1}^{K} \mathrm{C}_{k}^{\mathrm{ach}}$.

Although the BS cannot obtain the accurate channel estimation under pilot contamination as shown in (7), we expand $\hat{\mathbf{h}}_{k}^{\mathrm{H}}$ as (9b). This is to provide the theory gist for our pilot assignment algorithm in the next section. In (9b), $\Delta_{1}$ denotes the desired data sequence. $\Delta_{2}$ denotes the intra-cell interference caused by the spatial correlation between distinct channel response vectors. $\Delta_{3}$ and $\Delta_{4}$ denote the intra-cell interference caused by the pilot reuse (i.e., pilot contamination). $\mathbf{n} \in C^{1 \times \kappa}$ denotes the equivalent data noise, and it can be expressed as $\mathbf{n}=\left(\hat{\mathbf{h}}_{k}-\mathbf{N}_{\mathrm{p}} \mathbf{z}_{k}^{\mathrm{H}}\right)^{\mathrm{H}} \mathbf{N}_{\mathrm{d}}+\mathbf{z}_{k} \mathbf{N}_{\mathrm{p}}^{\mathrm{H}} \mathbf{R}$. According to the state-of-the-art researches [16][17], we have $\frac{\left|\mathbf{n n}^{\mathrm{H}}\right|}{M} \stackrel{M \rightarrow \infty}{=} 0$.

According to (9b), the $k$-th user's uplink SINR can be calculated by

$$
\operatorname{SINR}_{k}=\frac{\left|\sqrt{\rho_{k}^{\mathrm{p}} \rho_{k}^{\mathrm{d}}} \mathbf{h}_{k}^{\mathrm{H}} \mathbf{h}_{k}\right|^{2}}{\sum_{j \neq k}^{K}\left|\sqrt{\rho_{k}^{\mathrm{p}} \rho_{j}^{\mathrm{d}}} \mathbf{h}_{k}^{\mathrm{H}} \mathbf{h}_{j}\right|^{2}+\sum_{i \in \Gamma_{k}}\left|\sqrt{\rho_{i}^{\mathrm{p}} \rho_{k}^{\mathrm{d}}} \mathbf{h}_{i}^{\mathrm{H}} \mathbf{h}_{k}\right|^{2}+\sum_{i \in \Gamma_{k}} \sum_{j \neq k}^{K}\left|\sqrt{\rho_{i}^{\mathrm{p}} \rho_{j}^{\mathrm{d}}} \mathbf{h}_{i}^{\mathrm{H}} \mathbf{h}_{j}\right|^{2}+\left|\mathbf{n} \mathbf{n}^{\mathrm{H}}\right|} \cdot
$$

In addition, if the amount of BS antennas is equal to infinity, the spatial correlation can be represented by large-scale fading variables and the AOA span as shown in (5). Therefore, 
when the BS antenna number $M \rightarrow \infty$, (11) can be rewritten as

$$
\operatorname{SINR}_{k}^{\infty}=\frac{\left|\sqrt{\rho_{k}^{\mathrm{p}} \rho_{k}^{\mathrm{d}}} \beta_{k} \theta_{k k}\right|^{2}}{\sum_{j \neq k}^{K}\left|\sqrt{\rho_{k}^{\mathrm{p}} \rho_{j}^{\mathrm{d}} \beta_{k} \beta_{j}} \theta_{k j}\right|^{2}+\sum_{i \in \Gamma_{k}}\left|\sqrt{\rho_{i}^{\mathrm{p}} \rho_{k}^{\mathrm{d}} \beta_{i} \beta_{k}} \theta_{i k}\right|^{2}+\sum_{i \in \Gamma_{k}} \sum_{j \neq k}^{K}\left|\sqrt{\rho_{i}^{\mathrm{p}} \rho_{j}^{\mathrm{d}} \beta_{i} \beta_{j}} \theta_{i j}\right|^{2}} .
$$

With sufficient orthogonal pilot sequences (i.e., $\tau \geq K$ ), we have $\Delta_{3}=0$ and $\Delta_{4}=0$ in (9b). However, $\Delta_{1}$ and $\Delta_{2}$ remain unchanged. Therefore, the $k$-th user's uplink SINR can be calculated as

$$
\operatorname{SINR}_{k}^{\text {noint }}=\frac{\left|\sqrt{\rho_{k}^{\mathrm{p}} \rho_{k}^{\mathrm{d}}} \mathbf{h}_{k}^{\mathrm{H}} \mathbf{h}_{k}\right|^{2}}{\sum_{j \neq k}^{K}\left|\sqrt{\rho_{k}^{\mathrm{p}} \rho_{j}^{\mathrm{d}}} \mathbf{h}_{k}^{\mathrm{H}} \mathbf{h}_{j}\right|^{2}+\left|\mathbf{n}_{\text {noint }} \mathbf{n}_{\text {noint }}^{\mathrm{H}}\right|} \stackrel{M \rightarrow \infty}{=} \frac{\left|\sqrt{\rho_{k}^{\mathrm{p}} \rho_{k}^{\mathrm{d}}} \beta_{k} \theta_{k k}\right|^{2}}{\sum_{j \neq k}^{K}\left|\sqrt{\rho_{k}^{\mathrm{p}} \rho_{j}^{\mathrm{d}} \beta_{k} \beta_{j}} \theta_{k j}\right|^{2}}
$$

In (13), $\mathbf{n}_{\text {noint }}=\left(\hat{\mathbf{h}}_{k}^{\text {noint }}-\mathbf{N}_{\mathrm{p}} \mathbf{z}_{k}^{\mathrm{H}}\right)^{\mathrm{H}} \mathbf{N}_{\mathrm{d}}+\mathbf{z}_{k} \mathbf{N}_{\mathrm{p}}^{\mathrm{H}} \mathbf{R}$. Similarly, we have $\frac{\left|\mathbf{n}_{\text {noint }} \mathbf{n}_{\text {noint }}^{\mathrm{H}}\right|}{M} \stackrel{M \rightarrow \infty}{=} 0$.

\section{Pilot Assignment}

In this section, the motivation of pilot assignment is presented firstly. Then a problem is designed to optimize the achievable system capacity. At last, a novel low-complexity pilot assignment algorithm is presented to solve the optimization problem.

\subsection{Motivation of Pilot Assignment}

As mentioned above, the typical massive MIMO systems can provide high-performance service with sufficient orthogonal pilot sequences. However, the amount of available orthogonal pilot sequences is limited because it is restricted by the length of the first part, i.e., pilot transmission part. In other words, the maximum number of users which can be served at the BSs simultaneously is limited with $\tau$ pilot symbols [6]. Therefore, pilot reuse problem is always inevitable in TDD massive MIMO systems.

With insufficient orthogonal pilot sequences (i.e., $\tau<K$ ), how to assign the limited orthogonal pilots to the terminals has an important influence on system performance. According to the analysis produced in Section 2, the achievable system capacity will be severely damaged if the orthogonal pilots are allocated to the users with low spatial correlations. On the other hand, a pilot assignment approach which allocates the orthogonal pilots to the users with high spatial correlations can make significant improvement in system performance. This conclusion motivates our pilot assignment algorithm.

\subsection{Problem Formulation}

In order to optimize the achievable system capacity, a problem is formulated as

$$
\max _{\{v\}} C_{\text {total }}^{\text {ach }} \text {. (14) }
$$

In (14), $\{v\}$ means all possible pilot sequence assignment approaches. Specifically, pilot assignment algorithm corresponding to the distinct channel AOA intervals is a research priority in this paper. Therefore, we discuss the limit of the achievable system capacity which is directly related to the incidence angle $\theta$ as

$$
\max _{\{0\}} C_{\text {total }}^{\infty} \cdot
$$


In (15), $\mathrm{C}_{\text {total }}^{\infty}=\sum_{k=1}^{K} \mathrm{C}_{k}^{\infty}$ and $\mathrm{C}_{k}^{\infty}=W \frac{(T-\tau)}{T} \log _{2}\left(1+\operatorname{SINR}_{k}^{\infty}\right)$. However, we find that it is difficult to evaluate $\operatorname{SINR}_{k}^{\infty}$ directly since $\operatorname{SINR}_{k}^{\infty}$ is associated with many factors as shown in (12), including the pilot transmission power, the data transmission power, the large-scale fading coefficient, as well as the span of the AOA intersection. Therefore, only the exhaustive search approach can be adopted to obtain the optimization solution to (15). This means we must explore all kinds of pilot assignment approaches and pick up the most optimal strategy. However, when the number of terminals is large, it is hard to implement the exhaustive search in practice because of its exponential complexity (i.e., $\tau^{K}$ ).

In the following discussion, we assume that a power control technology is adopted to compensate the large-scale fading coefficient, so that $\rho_{i}^{\mathrm{p}} \rho_{j}^{\mathrm{d}} \beta_{i} \beta_{j}$, for $i, j=1,2, \cdots, K$, can be regarded as a constant [18][19]. For a simple example, $\rho_{i}^{\mathrm{p}}=\rho_{i}^{\mathrm{d}}=\frac{\lambda}{\beta_{i}}$, for $i=1,2, \cdots, K$. This assumption is available and the reason is as follows. The fluctuations of the pilot transmission power and the data transmission power are very small because the average large-scale fading variables change very slowly in reality. Then (12) can be rewritten as

$$
\overline{\operatorname{SINR}}_{k}^{\infty} \sim \frac{\left|\theta_{k k}\right|^{2}}{\sum_{j \neq k}^{K}\left|\theta_{k j}\right|^{2}+\sum_{i \in \Gamma_{k}}\left|\theta_{i k}\right|^{2}+\sum_{i \in \Gamma_{k}} \sum_{j \neq k}^{K}\left|\theta_{i j}\right|^{2}} .
$$

In (16), denotes the symbol of "proportion to". Different from $\operatorname{SINR}_{k}^{\infty}, \overline{\operatorname{SINR}}_{k}^{\infty}$ can be easily evaluated because it is only related to the span of the AOA intersection. In addition, the optimization problem (15) can be simplified as

$$
\max _{\{\nu\}} \overline{\mathrm{C}}_{\text {total }}^{\infty}(17)
$$

In (17), $\quad \overline{\mathrm{C}}_{\text {total }}^{\infty}=\sum_{k=1}^{K} \overline{\mathrm{C}}_{k}^{\infty}$ and $\quad \overline{\mathrm{C}}_{k}^{\infty}=W \frac{(T-\tau)}{T} \log _{2}\left(1+\overline{\mathrm{SINR}}_{k}^{\infty}\right)$. Although there are two transformations from (14) to (17), the simulation results will verify that the pilot sequence assignment way which is proposed according to an analysis of (17) can approach the optimization solution to (14) well.

\subsection{Pilot Assignment Algorithm}

Firstly we need a parameter to measure the AOA similarity between two distinct terminals. Note that $\theta_{j i}$ in (5) is not adequate because it only can measure the AOA intersection between the $i$-th terminal and the $j$-th terminal. Therefore, we define a new parameter as

$$
\eta_{i j}=\frac{\theta_{i j}}{\delta_{i j}} \text {.(18) }
$$

In (18), $\delta_{i j}$ denotes the span of the AOA union, i.e., the span of $\left[\theta_{i}^{\min }, \theta_{i}^{\max }\right] \cup\left[\theta_{j}^{\min }, \theta_{j}^{\max }\right]$. In addition, $\eta_{i j} \in[0,1]$. Specifically, $\eta_{i j}=0$ denotes that the AOA for the $i$-th terminal and the AOA for the $j$-th terminal are non-overlapping, i.e., $\left[\theta_{i}^{\min }, \theta_{i}^{\max }\right] \cap\left[\theta_{j}^{\min }, \theta_{j}^{\max }\right]=\phi \cdot \eta_{i j}=1$ denotes that $\left[\theta_{i}^{\min }, \theta_{i}^{\max }\right]=\left[\theta_{j}^{\min }, \theta_{j}^{\max }\right]$. It is easy to understand that a higher value of $\eta_{i j}$ indicates a higher AOA similarity. Moreover, $\eta_{i j}$ inherits the advantage of $\theta_{i j}$ that the complex process of calculating the channel covariance matrices can be omitted. 
The pilot sequence assignment approaches mentioned in [11] and [12] indicated that the terminals with orthogonal channel covariance matrices can reuse the same pilot sequences. However, they certainly do not consider the different channel qualities. Specifically, after the terminals with "similar" channel covariance matrices are assigned with the orthogonal pilots, each unhandled user is matched up with the "best" pilot sequence disorderly.

Based on an analysis of the expression for $\bar{C}_{\text {total }}^{\infty}$, we can know: 1$)$. The orthogonal pilot signals should be allocated to the terminals with high AOA similarities. 2). The orthogonal pilot signals should be allocated to the terminals with large AOA spans. By this means, the intra-cell interference caused by the pilot reuse can be reduced as much as possible. Then the achievable system capacity can be improved significantly.

Based on this fact, a pilot assignment algorithm which considers the different channel qualities is proposed. Specifically, the proposed assignment approach mainly consists of three steps. Firstly, $\tau$ terminals with high AOA similarities are selected and the orthogonal pilot signals are allocated to them respectively. Then the remainder $(K-\tau)$ terminals are sorted in descending order according to their own AOA spans. At last, each one of these terminals is assigned with the most "appropriate" pilot sequence in a sequential way.

As mentioned above, $\eta_{i j}$ is adopted to measure the AOA similarity and $\theta_{k k}$ is adopted to measure the AOA span for the $k$-th terminal. In addition, let $\Omega_{\mathrm{UE}}=\left\{\Omega_{1}, \Omega_{2}, \cdots, \Omega_{K}\right\}$ denotes the terminal set. $G_{k}$, for $k=1,2, \cdots, \tau$, denotes the set of the terminals which share the $k$-th pilot sequence. And $A_{k}$ denotes the corresponding AOA union associated with the terminals in $G_{k}$. The specific pilot assignment method is presented in Algorithm 1.

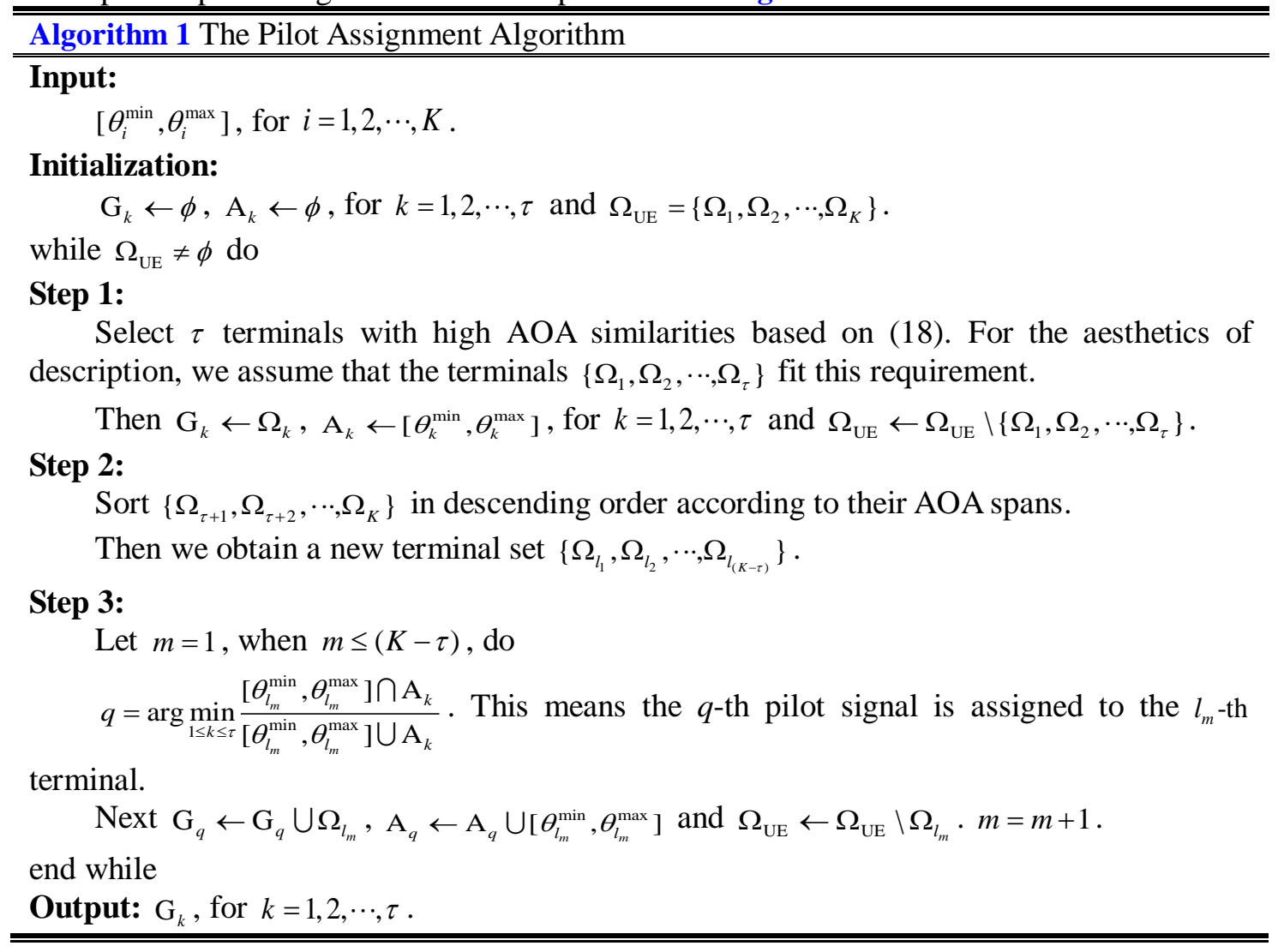


Note that for the proposed approach, we assume that the individual channel AOA intervals of all terminals can be calculated separately. This is valid in practical wireless communication networks [20]. In addition, similar to the method used in [11], this assumption can also be done by using the resource blocks (RBs) where the desired terminal and the interference terminals are allocated in different times. Next, the complexity of this assignment algorithm is evaluated. For step 1 , the computational complexity of getting all the parameters $\eta_{i j}$, for $i, j=1,2, \cdots, K$, is $O\left(K^{2}\right)$. Then the quicksort approach is adopted to select the eligible terminals and the complexity is $O\left(K^{2} \log _{2} K^{2}\right)$. For step 2, the computational complexity of getting the AOA spans for the remainder terminals is $O(K-\tau)$. Then the quicksort approach is adopted and the complexity is $O\left((K-\tau) \log _{2}(K-\tau)\right)$. For step 3, the computational complexity is $O((K-\tau) \tau)$. Therefore, the complexity of this assignment algorithm is $O\left(K^{2} \log _{2} K^{2}\right)$. When the number of terminals is large, it is obvious that the pilot assignment method mentioned above has the ability to reduce the complexity significantly compared with the exhaustive search.

In addition, because the single-cell massive MIMO model is adopted in [12], we take the pilot assignment approach mentioned in this paper as an example. The computational complexity of running the pilot assignment mentioned in [12] is $O\left(M^{2} K^{3}\right)$, which is proportional to the BS antenna number. This is because the individual covariance matrices of all users must be calculated separately for the pilot assignment approaches mentioned in [11] and [12]. However, the computational complexity of the proposed assignment algorithm is independent of the amount of BS antennas. This means the complex process of calculating the channel covariance matrices can be omitted by using the proposed parameter to measure the AOA similarity between two distinct terminals. It is obviously that $O\left(M^{2} K^{3}\right)>>\left(K^{2} \log _{2} K^{2}\right)$ for massive MIMO circumstances where the amount of BS antennas is usually very large.

\section{Simulation Results}

Firstly, we evaluate the channel estimation error performance and the achievable system capacity performance through Monte-Carlo simulations in this section. Specifically, the average large-scale fading coefficient of the $k$-th user $\beta_{k}$ is modeled as

$$
\beta_{k}=\frac{z_{k}}{r_{k}^{\gamma}} \text {. }
$$

In (19), $r_{k}$ refers to the average geographical distance between BS antennas and the $k$-th user. $\gamma$ denotes the decay exponent based on the experience. In addition, the value of $10 \log \left(z_{k}\right)$ follows $C N\left(0, \zeta^{2}\right)$ distribution.

As shown in Fig. 1, we hold the opinion that all terminals in the cell are randomly located without the radius of $r$. And the $k$-th user's channel AOA interval is denoted by $\left[\bar{\theta}_{k}-\left|\theta_{k}^{\Delta}\right|, \bar{\theta}_{k}+\left|\theta_{k}^{\Delta}\right|\right] \in\left[-\frac{\pi}{2}, \frac{\pi}{2}\right]$. Specifically, $\bar{\theta}_{k}$ denotes the average channel AOA for the $k$-th terminal, and we assume that it is uniformly distributed in the angle interval $\left[-\frac{\pi}{4}, \frac{\pi}{4}\right]$ in radians. In addition, we assume that $\theta_{k}^{\Delta}$, for $k=1,2, \cdots, K$, is $C N\left(0, \sigma^{2}\right)$ distributed. Other simulation parameters are summarized in Table $\mathbf{1}$.

In our simulations, the assignment method [11][12] denotes that the terminals with orthogonal channel covariance matrices reuse the same pilot sequences. Specifically, to obtain 
the channel covariance matrices of the terminals, we think that uniform linear array (ULA) is installed at the BS and the antenna spacing equals to a half wavelength distance. The random assignment denotes that the available pilot sequences are assigned to the terminals randomly.

Table 1. Simulation Parameters

\begin{tabular}{|c|c|}
\hline Number of terminals in the cell $K$ & 10 \\
\hline Radius of hexagonal cell $R$ & $200 \mathrm{~m}$ \\
\hline Radius of disk $r$ & $100 \mathrm{~m}$ \\
\hline Total bandwidth $W$ & $20 \mathrm{MHZ}$ \\
\hline Data SNR & $10 \mathrm{~dB}$ \\
\hline Decay exponent $\gamma$ & 3.8 \\
\hline Log normal shadowing fading factor $\zeta$ & $8 \mathrm{~dB}$ \\
\hline
\end{tabular}

\subsection{Channel Estimation Error Comparison}

In this subsection, the channel estimation error performance is evaluated through a defined parameter called normalized channel estimation error. Specifically, it can be expressed as

$$
10 \log _{10}\left(\frac{\sum_{k=1}^{K}\left|\left(\hat{\mathbf{h}}_{k}-\mathbf{h}_{k}\right)^{\mathrm{H}}\left(\hat{\mathbf{h}}_{k}-\mathbf{h}_{k}\right)\right|}{\sum_{k=1}^{K}\left|\mathbf{h}_{k}^{\mathrm{H}} \mathbf{h}_{k}\right|}\right) \text {. }
$$

The optimization solution denotes the minimum normalized channel estimation error obtained by the exhaustive search. In addition, we assume that $T=20$ symbols and $M=128$.

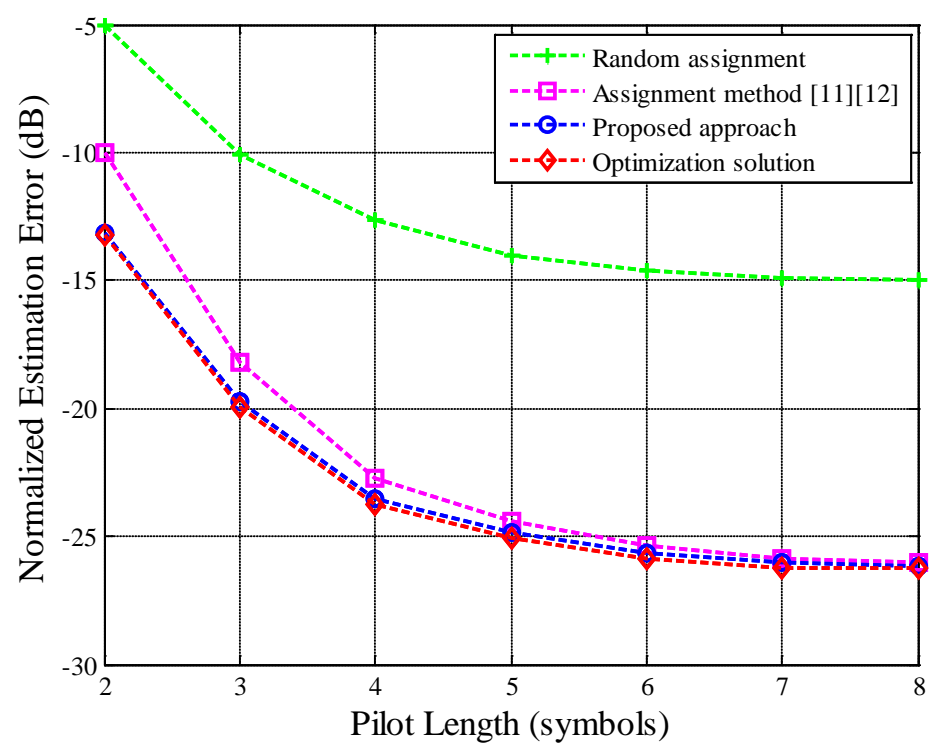

Fig. 2. Normalized channel estimation error vs. pilot length $\tau$ : pilot $\mathrm{SNR}=-5 \mathrm{~dB}$ and $\sigma=60^{\circ}$.

Fig. 2 reveals the normalized channel estimation error metric in (20) versus the pilot length $\tau$. It can be observed that no matter which kind of pilot assignment algorithm, the channel estimation error performance descends with the increase of pilot length. The proposed approach outperforms the existing method [11][12], especially in the short pilot length regime. In contrast, when $\tau \geq 7$, the performance gain obtained by using the proposed approach can be neglected. This is because a longer pilot length means a larger amount of available orthogonal 
pilot signals, and the benefit of exploiting new assignment algorithms in reducing the estimation error is not so obvious with sufficient orthogonal pilot sequences.

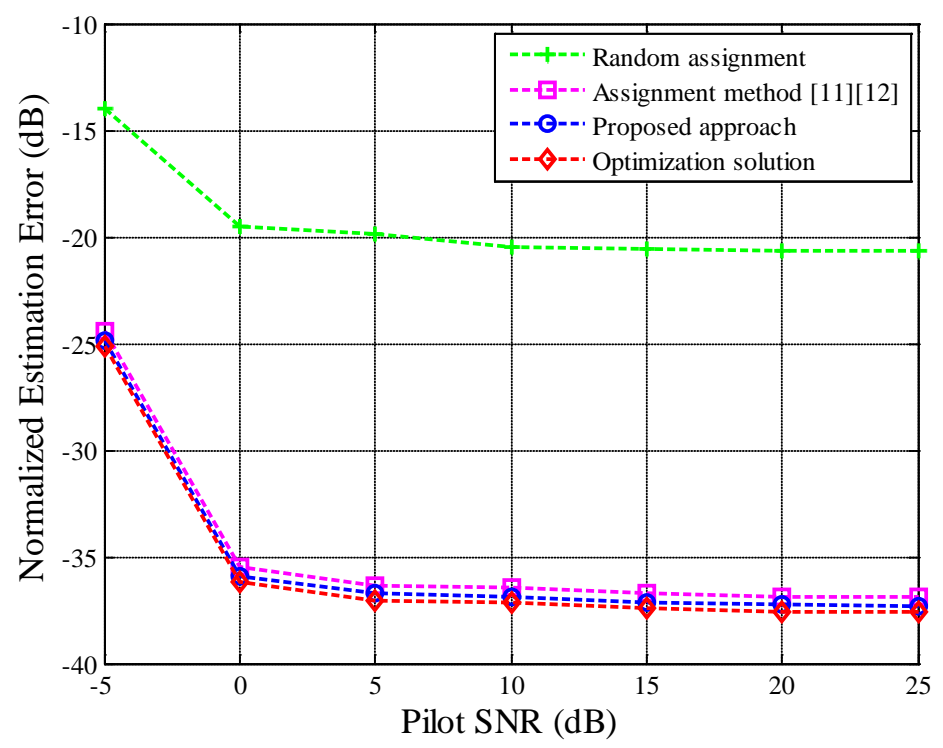

Fig. 3. Normalized channel estimation error vs. pilot SNR: $\tau=5$ symbols and $\sigma=60^{\circ}$.

Fig. 3 shows the estimation error metric versus the pilot SNR. In this figure, the channel estimation error performance descends with the increase of pilot SNR. Specifically, during the stage of SNR $\leq 5 \mathrm{~dB}$, the channel estimation error decreases significantly as the pilot SNR increases. For example, the proposed approach can provide a significant gain, for about $12 \mathrm{~dB}$, from pilot $\mathrm{SNR}=-5 \mathrm{~dB}$ to pilot $\mathrm{SNR}=5 \mathrm{~dB}$. However, during the stage of $\mathrm{SNR} \geq 5 \mathrm{~dB}$, the normalized estimation error remains nearly unchanged over a wide pilot SNR region.

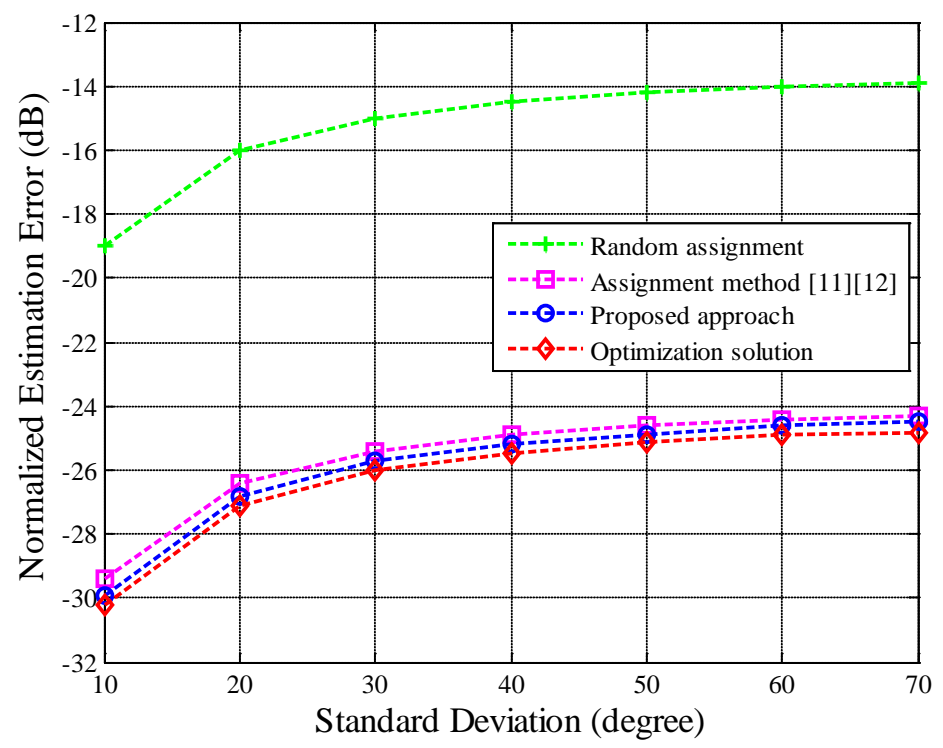

Fig. 4. Normalized channel estimation error vs. standard deviation $\sigma: \tau=5$ symbols and pilot $\mathrm{SNR}=-5 \mathrm{~dB}$. 
Fig. 4 shows the estimation error metric versus the standard deviation $\sigma$. This figure shows that the channel estimation error performance increases with the increase of standard deviation. Compared with the assignment method [11][12], the proposed approach is more sensitive to changes in standard deviation. For the former, the normalized estimation error increases from $-29.3 \mathrm{~dB}$ to $-24.3 \mathrm{~dB}$ when $\sigma$ increases from $10^{\circ}$ to $70^{\circ}$. On the other hand, the estimation error increases from $-30.0 \mathrm{~dB}$ to $-24.5 \mathrm{~dB}$ for the latter.

From Fig. 2 to Fig. 4, we can also obtain the following observations: 1). For the normalized channel estimation error metric, the performance of the main approach in this paper closely approaches that of the exhaustive search for different values of pilot length (pilot SNR and standard deviation). 2). The random assignment has the worst estimation error performance because it suffers from the most severe intra-cell interference.

\subsection{Achievable System Capacity Comparison}

In this subsection, the optimization solution denotes the solution to (14) that achieved by adopting exhaustive search method. We assume that $\tau=5$ symbols and pilot SNR $=10 \mathrm{~dB}$.

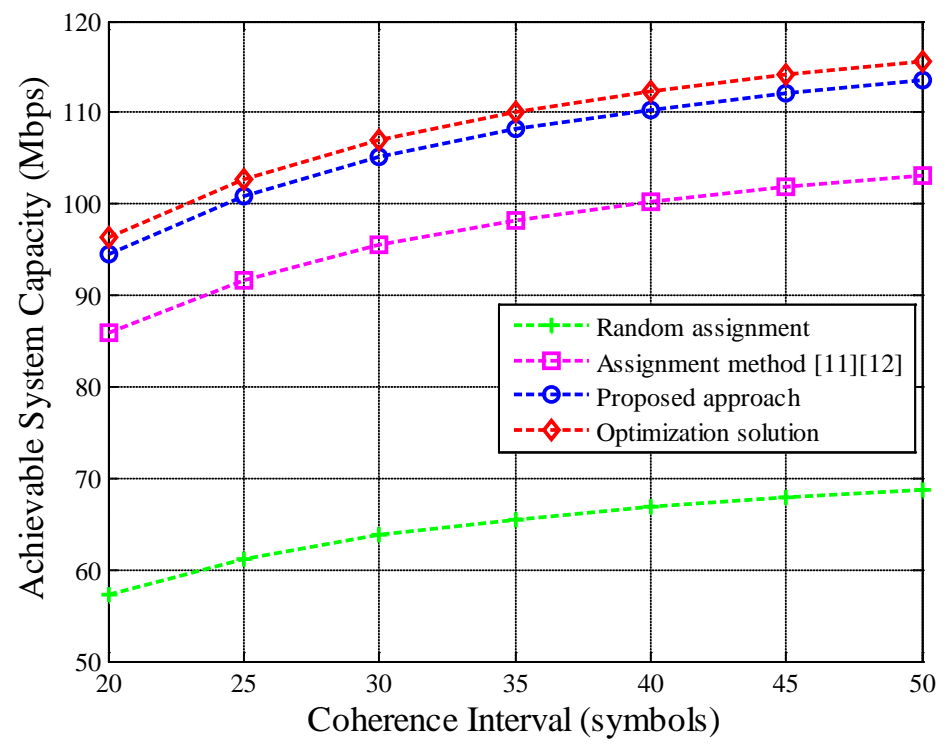

Fig. 5. Achievable system capacity vs. coherence interval $T: M=128$ and $\sigma=10^{\circ}$.

Fig. 5 shows the achievable system capacity metric $C_{\text {total }}^{\text {ach }}$ versus the coherence interval $T$. It can be observed that no matter which kind of pilot assignment algorithm, the achievable system capacity increases with the increase of coherence interval. Moreover, compared with the assignment method [11][12], the proposed approach provides a more significant performance gain in the long coherence interval regime. Specifically, when $T=20$ symbols, the gain is about $8 \mathrm{Mbps}$. And when $T=50$ symbols, the gain is about $11 \mathrm{Mbps}$. 


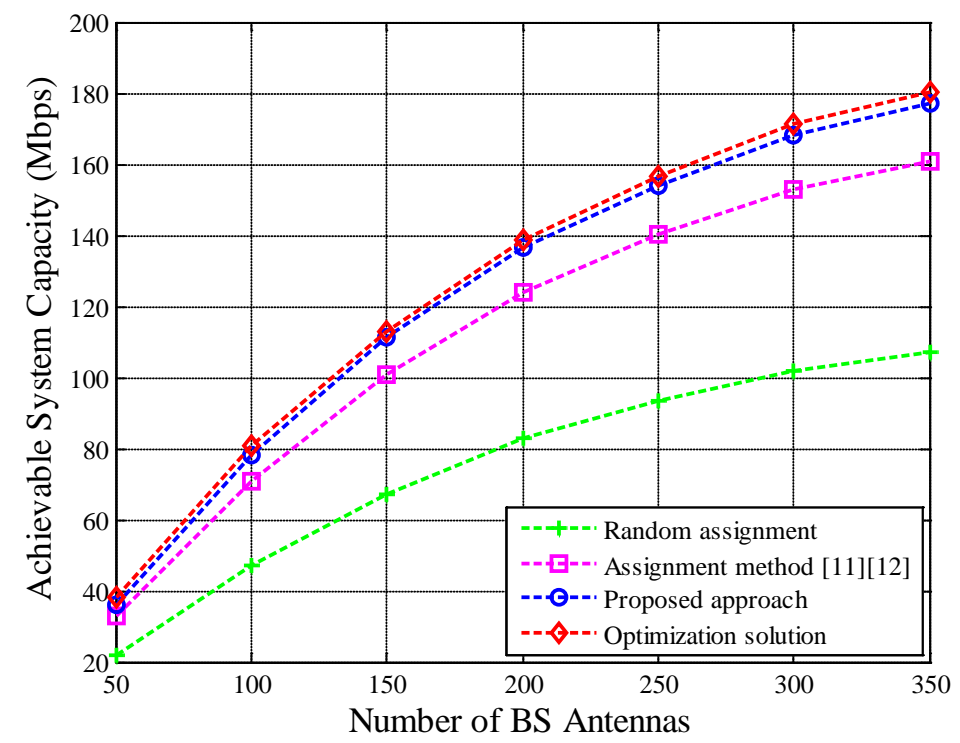

Fig. 6. Achievable system capacity vs. number of BS antennas $M: T=20$ symbols and $\sigma=10^{\circ}$.

Fig. 6 shows the system capacity metric versus the number of BS antennas $M$. In this fifure, the achievable system capacity increases with the increase of number of BS antennas. On the other hand, the proposed approach shows a performance gain over the assignment method [11][12], and the gain becomes larger as the BS antenna number becomes larger.

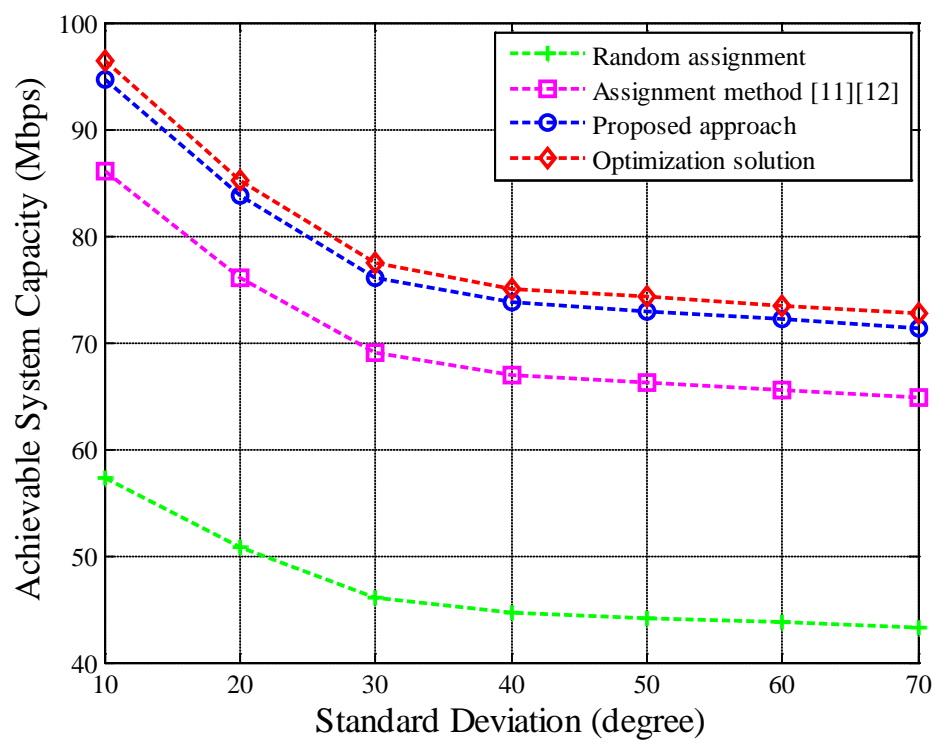

Fig. 7. Achievable system capacity vs. standard deviation $\sigma: T=20$ symbols and $M=128$.

Fig. 7 shows the system capacity metric versus the standard deviation $\sigma$. This figure shows that the achievable system capacity decreases with the increase of standard deviation. Specifically, when $\sigma \leq 30^{\circ}$, the system capacity decreases significantly as the standard deviation increases. Because in this range, the intra-cell interference caused by the spatial 
correlation and the pilot reuse is sensitive to changes in standard deviation. However, when $\sigma \geq 30^{\circ}$, the rate of capacity decrease slows down considerably.

From Fig. 5 to Fig. 7, we can also draw the following conclusions: 1). For the achievable system capacity metric, the performance of the main approach in this paper closely approaches that of the exhaustive search for different values of coherence interval (number of BS antennas and standard deviation). 2). The random assignment has the worst system capacity performance.

\section{Conclusion}

The pilot signal assignment is studied in single-cell multi-terminal TDD massive MIMO scenes with spatially correlated fading channels in this paper. We exploited the fact that the BS steering vectors associated with distinct incidence angles are orthogonal if the number of BS antennas is equal to infinity. Then the spatial correlation between two channel response vectors was established by the large-scale fading variables and the AOA span. With this model, the expression for the achievable system capacity was derived. Next, the optimization problem regarding uplink pilot assignment was designed to optimize the achievable system capacity. For the sake of approaching the optimization solution, the pilot sequence assignment algorithm corresponding to the distinct channel AOA intervals was proposed. Simulation results proved that the main pilot assignment algorithm in this paper can obtain a noticeable performance gain with limited BS antennas.

\section{References}

[1] W. Roh, J.-Y. Seol, J. Park, B. Lee, J. Lee, Y. Kim, J. Cho, K. Cheun, and F. Aryanfar, "Millimeter-wave beamforming as an enabling technology for 5G cellular communications: Theoretical feasibility and prototype results,” IEEE Communications Magazine, vol. 52, no. 2, pp. 106 - 113, February, 2014. Article (CrossRef Link)

[2] M. Shafi, A. F. Molisch, P. J. Smith, T. Haustein, P. Zhu, P. De Silva, F. Tufvesson, A. Benjebbour, and G. Wunder, "5G: A tutorial overview of standards, trials, challenges, deployment, and practice,” IEEE Journal on Selected Areas in Communications, vol. 35, no. 6, pp. 1201 - 1221, June, 2017. Article (CrossRef Link)

[3] E. Larsson, O. Edfors, F. Tufvesson, and T. Marzetta, "Massive MIMO for next generation wireless systems,” IEEE Communications Magazine, vol. 52, no. 2, pp. 186 - 195, February, 2014. Article (CrossRef Link)

[4] D. C. Araújo, T. Maksymyuk, A. L. de Almeida, T. Maciel, J. C. Mota, and M. Jo, "Massive MIMO: survey and future research topics,” IET Communications, vol. 10, no. 15, pp. 1938 - 1946, October, 2016. Article (CrossRef Link)

[5] J. Jose, A. Ashikhmin, T. L. Marzetta, and S. Vishwanath, "Pilot contamination problem in multi-cell TDD systems,” in Proc. of IEEE International Symposium on Information Theory (ISIT), Seoul, pp. 2184 - 2188, June 28 - July 3, 2009. Article (CrossRef Link)

[6] T. L. Marzetta, "Noncooperative cellular wireless with unlimited numbers of base station antennas,” IEEE Transactions on Wireless Communications, vol. 9, no. 11, pp. 3590 - 3600, November, 2010. Article (CrossRef Link)

[7] B. Han, S. Zhao, B. Yang, H. Zhang, P. Chen, and F. Yang, "Historical PMI based multi-user scheduling for FDD massive MIMO systems," in Proc. of IEEE 83rd Vehicular Technology Conference (VTC Spring), Nanjing, pp. 1 - 5, May 15 - 18, 2016. Article (CrossRef Link)

[8] X. Zhu, Z. Wang, L. Dai, and C. Qian, “Smart pilot assignment for massive MIMO,” IEEE Communications Letters, vol. 19, no. 9, pp. 1644 - 1647, September, 2015.

$\underline{\text { Article (CrossRef Link) }}$ 
[9] H. Zhang, S. Gao, D. Li, H. Chen, and L. Yang, "On superimposed pilot for channel estimation in multicell multiuser MIMO uplink: Large system analysis," IEEE Transactions on Vehicular Technology, vol. 65, no. 3, pp. 1492 - 1505, March, 2016. Article (CrossRef Link)

[10] R. R. Mueller, M. Vehkaperae, and L. Cottatellucci, "Blind pilot decontamination," IEEE Journal of Selected Topics in Signal Processing, vol. 8, no. 5, pp. 1016 - 1020, October, 2013. Article (CrossRef Link)

[11] H. Yin, D. Gesbert, M. Filippou, and Y. Liu, "A coordinated approach to channel estimation in large-scale multiple-antenna systems,” IEEE Journal on Selected Areas in Communications, vol. 31, no. 2, pp. 264 - 273, February, 2012. Article (CrossRef Link)

[12] L. You, X. Gao, X.-G. Xia, N. Ma, and Y. Peng, "Pilot reuse for massive MIMO transmission over spatially correlated rayleigh fading channels," IEEE Transactions on Wireless Communications, vol. 14, no. 6, pp. 3352 - 3366, February, 2015. Article (CrossRef Link)

[13] X. Zhu, L. Dai, and Z. Wang, "Graph coloring based pilot allocation to mitigate pilot contamination for multi-cell massive MIMO systems," IEEE Communications Letters, vol. 19, no. 10, pp. $1842-1845$, October, 2015. Article (CrossRef Link)

[14] X. Zhu, L. Dai, Z. Wang, and X. Wang, "Weighted-graph-coloring-based pilot decontamination for multicell massive MIMO systems," IEEE Transactions on Vehicular Technology, vol. 66, no. 3, pp. 2829 - 2834, March, 2017. Article (CrossRef Link)

[15] J. Jose, A. Ashikhmin, T. L. Marzetta, and S. Vishwanath, "Pilot contamination and precoding in multi-cell TDD systems," IEEE Transactions on Wireless Communications, vol. 10, no. 8, pp. 2640 - 2651, August, 2011. Article (CrossRef Link)

[16] F. Rusek, D. Persson, B. K. Lau, E. G. Larsson, T. L. Marzetta, O. Edfors, and F. Tufvesson, "Scaling up MIMO: Opportunities and challenges with very large arrays," IEEE Signal Processing Magazine, vol. 30, no. 1, pp. 40 - 60, January, 2013. Article (CrossRef Link)

[17] J. Hoydis, S. Ten Brink, and M. Debbah, "Massive MIMO in the UL/DL of cellular networks: How many antennas do we need?” IEEE Journal on Selected Areas in Communications, vol. 31, no. 2, pp. 160 - 171, January, 2013. Article (CrossRef Link)

[18] Q. Zhang, S. Jin, D. Morales, M. McKay, and H. Zhu, "Optimal pilot length for uplink massive MIMO systems with pilot reuse," in Proc. of IEEE International Conference on Acoustics, Speech and Signal Processing (ICASSP), Shanghai, pp. 3536 - 3540, March 20 - 25, 2016. Article (CrossRef Link)

[19] Q. Zhang, S. Jin, K.-K. Wong, H. Zhu, and M. Matthaiou, "Power scaling of uplink massive MIMO systems with arbitrary-rank channel means," IEEE Journal of Selected Topics in Signal Processing, vol. 8, no. 5, pp. 966 - 981, May, 2014. Article (CrossRef Link)

[20] H. Echigo, T. Ohtsuki, W. Jiang, and Y. Takatori, "Fair pilot assignment based on AOA and pathloss with location information in massive MIMO," in Proc. of IEEE Global Communications Conference (GLOBECOM), Singapore, pp. 1 -6, December 4 - 8, 2017. Article (CrossRef Link) 

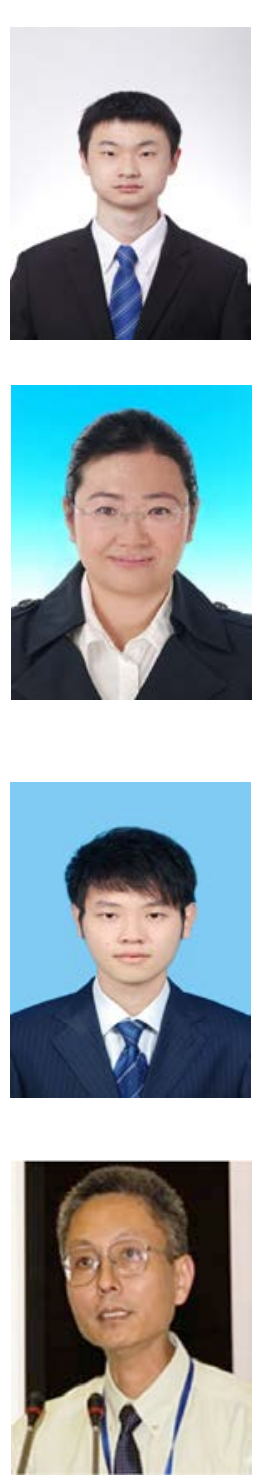

Pengxiang Li received his bachelor degree from Beijing University of Posts and Telecommunications (BUPT), Beijing, in 2014. He is currently pursuing the Ph.D. degree with the Wireless Theories and Technologies Lab, BUPT. His research interests include channel estimation, precoding, and signal design in massive MIMO.

Yuehong Gao received her BEng degree in Telecommunication Engineering from BUPT in 2004, PhD degree in Communication and Information System from BUPT in 2010, and $\mathrm{PhD}$ degree in Information and Communications Technology from Norwegian University of Science and Technology in 2012. Now she works in School of Information and Communication Engineering at BUPT as an associate professor. Her research interests include quality of service guarantees in communication networks, performance evaluation of wireless communication systems and system level simulation methodologies, with special emphasis on cognitive radio networks, 3G/4G/5G networks and heterogeneous networks related topics. She has published more than 40 academic papers in international conference and journals and applied more than 10 patents.

Zhidu Li received his bachelor degree from BUPT, Beijing, in 2012. He is currently pursuing the Ph.D. degree with the Wireless Theories and Technologies Lab, BUPT. His research interests include performance analysis of communication networks and application of network calculus.

Dacheng Yang received the Ph.D. degrees in communication and information system from BUPT, Beijing, China, in 1988. He has been a faculty member of School of Information and Telecommunications Engineering at BUPT since 1988, where he is currently the director and professor of Wireless Theories and Technologies Lab. Prof. Yang is doing research on the topics of Mobile Communication Technologies, Deployment and Application of Mobile Communication Systems, Standardization of Mobile Communication Systems. 